\title{
Power Control for Cellular Communications with Channel Uncertainties
}

\author{
S. Subramanian ${ }^{\dagger}$, J.M. Shea ${ }^{\dagger}$, and W.E. Dixon ${ }^{\dagger \ddagger}$ \\ Email:sankrith@ufl.edu,jshea@ece.ufl.edu,wdixon@ufl.edu \\ ${ }^{\dagger}$ Department of Electrical and Computer Engineering \\ ${ }_{\ddagger}^{\ddagger}$ Department of Mechanical and Aerospace Engineering \\ University of Florida, Gainesville, FL 32611
}

\begin{abstract}
In the reverse link of a wireless cellular network, power control is used to ensure that each link achieves its target signal-to-interference-plus-noise ratio (SINR), while minimizing the interference to adjacent cells. In cellular systems using direct-sequence code-division multiple access (CDMA), the SINR depends inversely on the power assigned to the other users in the system, creating a nonlinear control problem. Mobility of the terminals, along with associated random shadowing and multi-path fading, results in uncertainty in the channel state. A regulation controller is developed in this paper for a CDMA cellular system with uncertainties in the state and channel noise. The developed controller regulates the SINR to a small region about a target value. An analysis is also provided to examine how mobility and the desired SINR regulation range affects the choice of channel update times.
\end{abstract}

\section{INTRODUCTION ${ }^{1}$}

Various transmitter power control methods have been developed to deliver a desired quality of service (QoS) in wireless networks [1]-[8]. Early work on power control using a centralized approach was investigated in [9] and [10]. The concept of Signal-to-Interference (SIR) balancing was introduced in [9] and [10], where all receivers experience the same SIR levels. Maximum achievable SIRs were formulated considering the SIR balancing problem as an eigenvalue problem. Efforts were made to reduce co-channel interference for a given channel allocation using transmitter power control in [1] and [3]. In [1], performance is analyzed in terms of outage probabilities. A stochastic distributed transmitter power approach was also investigated in [1]-[3]. A simple distributed autonomous power control algorithm was introduced in [4] where channel reuse is maximized. A generalized framework for uplink power control is provided in [6], where common properties for interference constraints are identified. An upper limit for the power was imposed to each user in the constrained power control algorithm of [5]. Active link protection (ALP) schemes were introduced in [8] and [11], where the QoS of active links is maintained above a threshold limit to protect the link quality. Recently, a distributed power control (DPC) scheme was suggested in [12] in the presence of radio channel uncertainties caused by mobility of the user terminals. These channel uncertainties include exponential path loss, shadowing, and multi-path

\footnotetext{
${ }^{1}$ This research is supported in part by the Department of Energy URPR program grant number DE-FG04-86NE37967 and the National Science Foundation under grant number CNS-0626863.
}

fading, which are modeled as random variables in the SINR measurements.

Of the channel uncertainties, multi-path fading has the most critical effect on the design of a power-control system because of the time and amplitude scales. Multi-path fading is caused by reflections in the environment, which cause multiple time-delayed versions of the transmitted signal to add together at the receiver. The time offsets cause the signals to add with different phases, and thus multi-path fading can change significantly over distance scales as short as a fraction of a wavelength. For instance, for a system using the $900 \mathrm{MHz}$ cellular band, the channel coherence time (the time for which the channel is essentially invariant) for a mobile terminal traveling at $30 \mathrm{miles} / \mathrm{hour}$ is approximately $10 \mathrm{~ms}$. There is a need to quantify the multi-path fading effects of the channel in the system.

The uncertainty of the multi-path fading effects provided motivation for the results in [12] and [13]. Specifically, a persistently exciting adaptation scheme is proposed in [12] and [13]. However, in these works, the fading process is modeled as slowly changing so that the channel gain can be accurately estimated and practical limitations of transmission power limitations are not considered.

In this paper, we design and analyze the performance of a controller for use in a channel with Rayleigh fading following Clarke's model [14]. The Rayleigh fading process produces unbounded changes in the SINRs with non-zero probability, even for arbitrarily short time scales, but by using the concept of overfaded users [15], we can bound the channel gains. Based on this model, we develop a simple proportional controller to minimize the sampled SINR error. Specifically, despite uncertainty in the multi-path fading effects, a Lyapunov-based analysis is used to develop an ultimate bound for the sampled SINR error which is a function of the upper bound on the channel uncertainty divided by a nonlinear damping gain that can be made arbitrarily large up to some upper value dictated by the power update law. We evaluate the performance of this controller via simulation under realistic power limits and channel changes based on the standard random-waypoint mobility model. We also consider a statistical analysis of the performance effects of fading between the sampling intervals, which we use to discuss the choice of the control update rate. 


\section{Network Model AND Properties}

We consider the reverse channel (from the mobile terminals (MTs) to the base station (BS)) and investigate control of the SINRs for the MTs via centralized power control. The same approach is equally applicable to the forward link. The SINRs at the BS depend on the power transmitted by each mobile and the path losses from the MTs to the BS [12]. Based on the state space-based approach of [11] and [16], a generic MIMO model can be constructed as (see the appendix)

$$
x(l+1)=\alpha(l, x) x(l)+u(l)+\xi(l, x) .
$$

In (1), $x(l+1) \in \mathbb{R}^{n}$ is the $\operatorname{SINR}, \xi(l, x) \in \mathbb{R}^{n}$ is the stochastic measurement noise assumed to be bounded by a constant $v, \alpha(l, x) \in \mathbb{R}^{n \times n}$ denotes the unknown, timevarying state-dependent diagonal matrix (since $\alpha_{i}(l, x)$ is a function of the state $x_{i}(l)$ as shown in the appendix) that is assumed to be upper bounded by a known positive constant. Here, $u(l) \in \mathbb{R}^{n}$ is an input that is expressed in terms of the power update law as

$$
P_{i}(l+1)=\frac{u_{i}(l)}{x_{i}(l)} P_{i}(l)+P_{i}(l) .
$$

The SINR at the radio link $i$, denoted by $x_{i}(l) \in \mathbb{R}$, can be

expressed as [17]

$$
x_{i}(l)=\frac{g_{i i}(l) P_{i}(l)}{I_{i}^{a}(l)},
$$

where $P_{i}(l)$ is the power from the MT $i$ to the BS, and $g_{i i}(l)$ is the channel gain from the MT $i$ to the BS. In (3), $I_{i}^{a}(l)$ is the interference-plus-noise power at the BS from other MT transmissions to the BS modeled as

$$
I_{i}^{a}(l)=\frac{I_{i}(l)}{a}=\frac{\sum_{j \neq i} g_{i j}(l) P_{j}(l)}{a}+\eta_{i}(l),
$$

where

$$
I_{i}(l)=\sum_{j \neq i} g_{i j}(l) P_{j}(l)+a \eta_{i}(l),
$$

$a$ is the spreading factor for the cellular system using CDMA, and $\eta_{i}(l)$ is the noise variance in the receiver. The channel gain $g_{i j}(l)$ in (5) denotes the gain in the radio link between the MT $j$ to the BS that is responsible for the interference in radio link $i$, and $P_{j}(l)$ is the power from the MT $j$ to the BS. The channel gain, $g_{i i}(l)$, is modeled as

$$
g_{i i}(l)=g_{d_{0}}\left(\frac{d_{i}(l)}{d_{0}}\right)^{-\kappa} 10^{0.1 \delta_{i}(l)}\left|X_{i}(l)\right|^{2},
$$

where $g_{d_{0}}$ is the near-field gain given by [18]

$$
g_{d_{0}}=\frac{G_{t} G_{r} \lambda^{2}}{(4 \pi)^{2} d_{0}^{2} L}, \quad d_{f} \leq d_{0} \leq d_{i}(l),
$$

where $G_{t}$ is the transmitter antenna gain, $G_{r}$ is the receiver antenna gain, $\lambda$ is the wavelength in meters, $L$ is the systemloss factor, $d_{0}$ is the distance between the transmitter and receiver antenna, and $d_{f}=6 m$ is the Fraunhofer distance.
The antenna gains $G_{t}$ and $G_{r}$ and the loss factor $L$ are assumed to be 1 without loss of generality.

The channel variations in (6) consist of three components. The term $\left(\frac{d_{i}(l)}{d_{0}}\right)^{-\kappa}$ is used to model the average path loss at distance $d_{i}(l)$ from MT $i$ to the BS, where $\kappa$ is the path-loss exponent, which typically takes values between two and five. The term $10^{0.1 \delta_{i}(l)}$ is used to model large-scale log-normal shadowing from buildings, terrain, or foliage, where $\delta_{i}(l)$ is a Gaussian random process. The term $\left|X_{i}(l)\right|^{2}$ is used to model Rayleigh fading. For analytical purposes, $X_{i}(t)$ is usually taken to be a complex-valued Gaussian random process, and thus $|X(t)|$ is a Rayleigh random variable for each $t$ when $E[X(t)]=0$, which corresponds to no line-of-sight path from the MT to the BS. The Gaussian random processes provide good models for the log-normal shadowing and Rayleigh fading over the most-probable range of reception. However, both of these processes are unbounded, which means that any received power level is possible. However, $g_{i i}$ cannot take arbitrarily large values in practice because the received power cannot exceed the transmitted power. Furthermore, a cellular system cannot practically transmit to overfaded users who are in very deep fades (i.e., when $g_{i i}$ close to zero) because doing so would require extremely large power at that user and the other users (because the power transmitted to each user causes interference at the other users) [15].

\section{Control Development}

\section{A. Control Objective}

Typically, the SINR should remain between two thresholds as

$$
\gamma_{\min } \leq x_{i} \leq \gamma_{\max }
$$

to achieve acceptable communication performance over the link while minimizing interference to adjacent cells [12], [13]. The control objective for the following development is to regulate the SINR to a target value for each channel, denoted by $\gamma \in \mathbb{R}^{n}$, while ensuring that the SINR remains between the specified lower and upper limits for each channel, as described in (8). To quantify the objective, a regulation error $e(l) \in \mathbb{R}^{n}$ is defined as

$$
e(l)=x(l)-\gamma .
$$

\section{B. Closed-loop Error System}

The first difference of the regulation error, denoted as $\Delta e(l) \in \mathbb{R}^{n}$, is defined as

$$
\begin{aligned}
\Delta e(l) & =e(l+1)-e(l)=x(l+1)-x(l) \\
& =\alpha(l, x) x(l)+u(l)+\xi(l, x) w(l, x)-x(l) .
\end{aligned}
$$

To facilitate the subsequent analysis, the expression in (10) is rewritten as

$$
\Delta e(l)=\chi(l, x)+\Omega(l, x)+u(l),
$$

where $\chi(l, x) \in \mathbb{R}^{n}$ denotes an auxiliary term defined as

$$
\chi(l, x)=\left(\alpha(l, x)-I^{n \times 1}\right) e(l),
$$


and $\Omega(l, x) \in \mathbb{R}^{n}$ is defined as

$$
\Omega(l, x)=\left(\alpha(l, x)-I^{n \times 1}\right) \gamma+\xi(l, x) w(l, x) .
$$

Motivation for introducing the auxiliary terms in (12) and (13) is to collect terms that have a common upper bound. Specifically, upper bounds for $\chi(l, x)$ and $\Omega(l, x)$ can be developed as (refer Section II)

$$
\|\chi(l, x)\| \leq c_{1}\|e(l)\| \text { and }\|\Omega(l, x)\| \leq c_{2},
$$

where $c_{1}, c_{2} \in \mathbb{R}$ denote known positive constants. Based on (11), (14), and the subsequent stability analysis, a proportional controller is designed as

$$
u(l) \triangleq-\left(c_{1}+k_{n}+k_{1}\right) e(l)
$$

where $c_{1}$ is introduced in (14), and $k_{1}, k_{n} \in \mathbb{R}$ denote positive control gains. Based on (2) and (15), the power update law is

$$
P_{i}(l+1)=\frac{-\left(c_{1}+k_{n}+k_{1}\right) e_{i}(l) P_{i}(l)}{\left(e_{i}(l)+\gamma\right)}+P_{i}(l),
$$

where the power update law is constrained so that $0<$ $P_{i}(l) \leq P_{\max }$, where $P_{\max }$ is a maximum power level. After substituting (15) into (11), the closed-loop error system for $e(l)$ can be determined as

$$
\Delta e(l)=\chi(l, x)+\Omega(l, x)-\left(c_{1}+k_{n}+k_{1}\right) e(l) .
$$

\section{Stability ANALYSis}

Theorem 1: The controller in (15) and (16) ensures that the SINR regulation error approaches an ultimate bound $\varepsilon\left(k_{n}, l_{0}\right) \in \mathbb{R}$ in the sense that

$$
\|e(l)\| \rightarrow \varepsilon\left(k_{n}, l_{0}\right) \text { as } l \rightarrow \infty,
$$

provided the bounds in (14) are valid.

Proof: Let $V(e, l): D \times[0, \infty) \rightarrow \mathbb{R}$ be a positive definite function defined as

$$
V(e, l)=\frac{1}{2} e^{T}(l) e(l),
$$

where $e(l)$ is defined as (9). After taking the first difference of (18), substituting (11) into the resulting expression, and then cancelling common terms, the following expression can be obtained:

$$
\begin{aligned}
\Delta V= & e^{T}(l) \chi(l, x)+e^{T}(l) \Omega(l, x) \\
& -\left(c_{1}+k_{n}+k_{1}\right) e^{T}(l) e(l) .
\end{aligned}
$$

By using (14), the expression in (19) can be upper bounded as

$$
\begin{aligned}
\Delta V \leq & c_{1}\|e(l)\|^{2}+c_{2}\|e(l)\| \\
& -\left(c_{1}+k_{n}+k_{1}\right)\|e(l)\|^{2} \\
\leq & c_{2}\|e(l)\|-k_{n}\|e(l)\|^{2}-k_{1}\|e(l)\|^{2} .
\end{aligned}
$$

After completing the squares on the first two terms in (20), the following upper bound can be developed

$$
\Delta V \leq-k_{1} V(e, l)+\frac{c_{2}^{2}}{4 k_{n}}
$$

Lemma 13.1 of [19] can now be invoked to conclude that

$$
V(e, l) \leq b^{l} V\left(e\left(l_{0}\right), l_{0}\right)+\left(\frac{1-b^{l}}{k_{1}}\right) \frac{c_{2}^{2}}{4 k_{n}},
$$

where

$$
b=1-k_{1},
$$

where $0<k_{1} \leq 1$. Based on (22), an upper bound for $e(l)$ can be developed as

$$
\|e(l)\|^{2} \leq b^{l}\left\|e\left(l_{0}\right)\right\|^{2}+\left(\frac{1-b^{l}}{k_{1}}\right) \frac{c_{2}^{2}}{4 k_{n}} .
$$

The ultimate bound in (23) asymptotically converges as

$$
\lim _{l \rightarrow \infty}\|e(l)\|^{2}=\frac{c_{2}^{2}}{4 k_{1} k_{n}} .
$$

From (24), the ultimate bound can be decreased by increasing $k_{n}$; however, the magnitude of $k_{n}$ is restricted by (16) and the constraint that $0<P_{i}(l) \leq P_{\max }$.

\section{ESTIMATION OF ERROR AT UNSAMPLED INSTANCES}

The developed controller operates at discrete times using a predefined sampling rate. The stability analysis in Section IV only proves that the controller can achieve arbitrarily low error at the sampling times. In this section, an approximate analysis of the error is provided between the sampling times, and the mean-squared error is shown to be bounded by a constant that depends on the time between samples.

Consider the performance for large $t$, such that the error magnitude satisfies $|e(l)|=|x(l)-\gamma|<\varepsilon$. Let $T_{s}$ denote the time between samples. Then the error for the signal from MT $i$ at time $t$, where $l T_{s}<t<(l+1) T_{s}$ is

$$
e_{i}(t)=x_{i}(t)-\gamma=\frac{g_{i i}(t) P_{i}(t)}{I_{i}^{a}(t)}-\gamma
$$

Letting $\Delta g_{i i}(t)=g_{i i}(t)-g_{i i}\left(l T_{s}\right)$ and using (5), the error can be written as

$$
\begin{aligned}
e_{i}(t) & =\frac{a\left[g_{i i}(l)+\Delta g_{i i}(t)\right] P_{i}(t)}{\sum_{j \neq i}\left[g_{i j}(l)+\Delta g_{i j}(t)\right] P_{j}(l)+a \eta_{i}(t)}-\gamma \\
& =\frac{a g_{i i}(l) P_{i}(t)+a \Delta g_{i i}(t) P_{i}(t)}{\sum_{j \neq i} g_{i j}(l) P_{j}(l)+\sum_{j \neq i} \Delta g_{i j}(t) P_{j}(l)+a \eta_{i}(t)}-\gamma .
\end{aligned}
$$

To facilitate the analysis, under the assumption of a large number of mobile stations operating in the current cell, the weak law of large numbers can be invoked to approximate the second term in the denominator as

$$
\begin{aligned}
\sum_{j \neq i} \Delta g_{i j}(t) P_{j}(l) & \approx \sum_{j \neq i} E\left[\Delta g_{i j}(t)\right] \\
& =0 .
\end{aligned}
$$

Thus, the magnitude of the error can be approximated as

$$
\begin{aligned}
\left|e_{i}(t)\right| \approx\left|\frac{a g_{i i}(l) P_{i}(t)+a \Delta g_{i i}(t) P_{i}(t)}{\sum_{j \neq i} g_{i j}(l) P_{j}(l)+a \eta_{i}(t)}-\gamma\right| \\
\Rightarrow\left|e_{i}(t)\right|<\left|\frac{a \Delta g_{i i}(t) P_{i}(t)}{\sum_{j \neq i} g_{i j}(l) P_{j}(l)+a \eta_{i}(t)}\right|+\varepsilon_{C T} .
\end{aligned}
$$


Noting that $E\left[\Delta g_{i i}(t)\right]=0$, the mean-squared error at time $t$ is bounded by

$$
E\left[e_{i}^{2}(t)\right]<\frac{a^{2} E\left[\Delta g_{i i}^{2}(t)\right] P_{i}^{2}(t)}{\left[\sum_{j \neq i} g_{i j}(l) P_{j}(l)+a \eta_{i}(t)\right]^{2}}+\varepsilon_{C T}^{2},
$$

where the expectation $E\left[\Delta g_{i i}^{2}(t)\right]$ is with respect to the random change in the fading $\Delta g_{i i}(t)$.

Let $R_{g}(\tau)$ be the autocorrelation function of the channel gain process. The expected value in (25) can be written as

$$
\begin{aligned}
E\left[\Delta g_{i i}^{2}(t)\right] & =E\left\{\left[g(t)-g\left(l T_{s}\right)\right]^{2}\right\} \\
& =E\left[g^{2}(t)\right]-2 E\left[g(t) g\left(l T_{s}\right)\right]+E\left[g^{2}\left(l T_{s}\right)\right] \\
& =2 R_{g}(0)-2 R_{g}(\tau),
\end{aligned}
$$

where $\tau=t-l T_{s}$. In most systems, the sampling time will be fast enough that the exponential path loss and shadowing can be modeled as constant between sampling times, and thus we consider only the effects of multi-path fading. The autocorrelation function for the power in a Rayleigh fading process is given by [14]

$$
R_{g}(\tau)=J_{0}^{2}\left(2 \pi f_{n} \tau\right)
$$

where $J_{0}$ is the zeroth-order Bessel function of the first kind, and $f_{n}$ is the Doppler spread. The Doppler spread is given by $f v / c$, where $f$ is the carrier frequency, $v$ is the mobile velocity, and $c$ is the speed of light. Then, the mean-squared error is bounded by

$$
\begin{aligned}
E\left[e_{i}^{2}(t)\right] & <\frac{2 a^{2}\left[1-J_{0}^{2}\left(2 \pi f_{n} \tau\right)\right] P_{i}^{2}(t)}{\left[\sum_{j \neq i} g_{i j}(l) P_{j}(l)+a \eta_{i}(t)\right]^{2}}+\varepsilon_{C T}^{2} \\
& <2 a^{2}\left[1-J_{0}^{2}\left(2 \pi f_{n} T_{s}\right)\right] \frac{P_{\max }^{2}}{n^{2} P_{\min }^{2}}+\varepsilon_{C T}^{2},
\end{aligned}
$$

where we apply the weak law of large numbers to the denominator with $E\left[g_{i j}^{2}(l)\right]=1$. Here, $P_{\max }$ and $P_{\min }$ are, respectively, the maximum and minimum transmit powers allocated to a non-overfaded user. By taking into account the maximum power ratio $P_{\max } / P_{\min }$, number of users $n$, spreading gain $a$, and maximum MT velocity, $T_{s}$ can be selected to make the mean-square error be arbitrarily close to $\varepsilon_{C T}$. However, since the mean-square error can never be identically 1 , it is not possible to obtain zero error convergence result for this discrete-time system.

To give an idea of the implication of (25), consider the error when $\varepsilon_{C T} \approx 0$. Let the carrier frequency $f=900 \mathrm{Mhz}$ and maximum velocity $v=30$ miles/hour. Then the Doppler spread is $40.2 \mathrm{~Hz}$. To achieve a maximum meansquare error of $0.1 S N R_{\max }$, where $S N R_{\max }=\frac{2 a^{2} P_{\max }^{2}}{n^{2} P_{\max }^{2}}$, the sampling time must be approximately $1.8 \mathrm{~ms}$. The ability to achieve this goal depends on the data rate in the system. For example, at $100 \mathrm{kbps}$ data rate, this requires a power control update every 178bits.

\section{Simulation Results}

A cellular network topology was built in MATLAB and the mobility of ten MTs is modelled by a steady state (stationary) distribution model (i.e., [20], [21]). A Random-Waypoint model is used to simulate the mobility of the MTs. The error signal is expressed as

$$
e_{i_{d B}}(l)=10 \log \left(\frac{x_{i}(l)}{\gamma}\right) d B,
$$

where $\gamma=8 d B$ is the target SINR as defined in Section II with a range between 6 and $10 d B$. Thermal noise, $\eta$, is set to $-110 d B m$. A Rayleigh faded channel is created using the channel sampling time of $1.7 \mathrm{~ms}$ obtained from the error analysis (Section V) and the Doppler frequency, given by [18]

$$
v=\frac{v \cos \theta}{\lambda},
$$

where $v$ is the actual velocity of the MT, $\theta$ is the angle between the BS and the direction of motion of the MT, and $\lambda=0.33 m$ is the wavelength of the signal. The probability density function of the velocity is given by [21]

$$
f_{i}(v)=\frac{C_{h}}{v} f_{V \mid h}^{0}(v)
$$

where

$$
\begin{aligned}
f_{V \mid h}^{0}(v) & =\frac{1}{v_{\max }-v_{\min }} \\
& =\frac{1}{48 k m / h r-2 k m / h r}=\frac{1}{46 k m / h r}
\end{aligned}
$$

is a classical choice for the density of the velocity, $C_{h}=$ 14.47 is the normalizing constant, and $v$ is the actual velocity of the MT. The subscript $h$ is used to denote the phase of the MT [21]. The velocity for each of the MTs is obtained from (28) using the inverse transform method as

$$
v=\exp (3.179 r+0.6931),
$$

where $r$ is uniformly distributed between 0 and 1 . The Doppler frequency is obtained from (30) and by measuring $\theta$ periodically. Path loss, with free space propagation effects (near-field effects), and log-normal shadowing are modelled [18] as shown in (6-7).

The results in Fig. 1-4 are obtained with

$$
c_{1}=8 \times 10^{-5}, \quad k_{1}=5 \times 10^{-5}, \quad k_{n}=1.625,
$$

and the spreading factor $a$ is chosen as 320. Figs. 1 and 2 depict the SINR errors for radio links operating at Doppler frequencies ranging from $0-10 \mathrm{~Hz}$ and from $10-35 \mathrm{~Hz}$, respectively. Figs. 3 and 4 depict the SINR error, channel uncertainty, and power transmission levels for Doppler frequencies of $1.98 \mathrm{~Hz}$ and $34.14 \mathrm{~Hz}$, respectively. These plots indicate the intuitive notion that the SINR error is within the desired threshold for more samples at lower Doppler frequencies that at the higher frequencies. The second column of Table I quantifies the percentage of samples that lie within the desired SINR range for each Doppler frequency. When samples exceed the upper limit of the desired SINR 


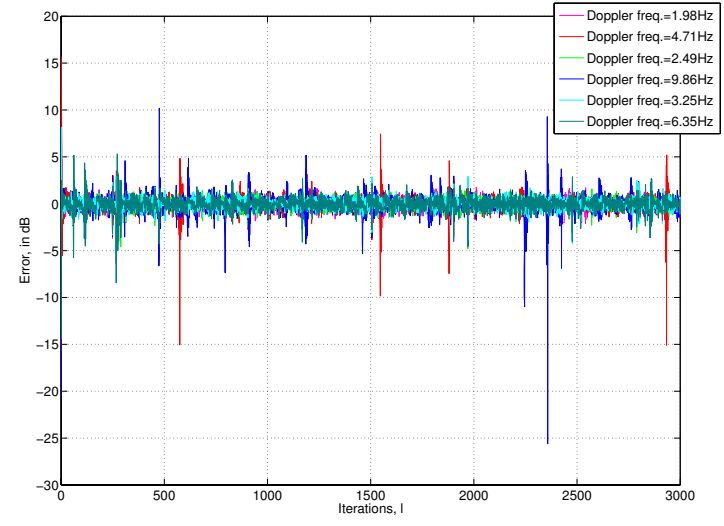

Fig. 1. Error Plot: MTs with low doppler frequencies

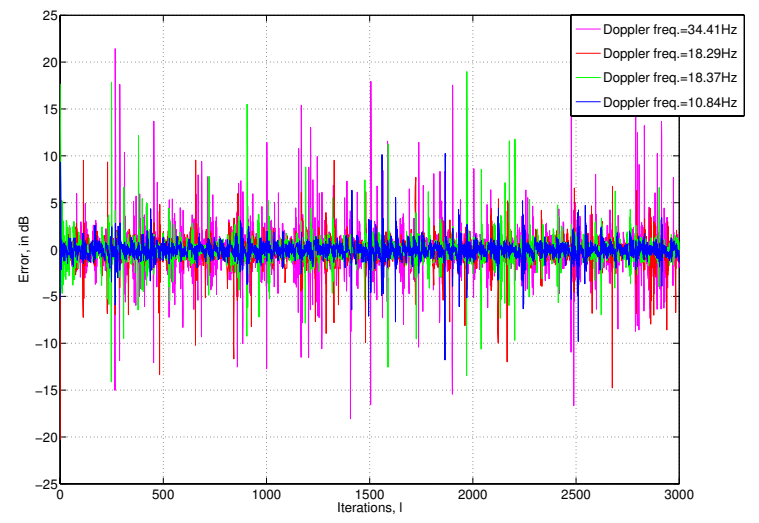

Fig. 2. Error Plot: MTs with high doppler frequencies

range (i.e., $x_{i}(l) \geq \gamma_{\max }$ ), the quality of service for the individual link is not compromised. However, exceeding the upper limit is undesirable because the interference to other links increases, potentially leading to an outage (i.e., when $\left.x_{i}(l) \leq \gamma_{\min }\right)$ [1]-[3]. An outage of a link does compromise the quality of service in the sense that the signal may not be decoded at that particular sample. The third column of Table I quantifies the percentage of samples that experience an outage for each Doppler frequency, particularly due to fading [22], [23].

Figs. 1-4 and Table I indicate that some samples fall outside of the desired SINR range (and experience an outage) with increasing occurrences at higher Doppler frequencies. The simulation model included a realistic upper limit on the available power (i.e., $27 \mathrm{dBm}(500 \mathrm{~mW})$ ) with a fixed sampling frequency. The simulation also yields rapid changes in the channel gains (i.e., high frequency components in the uncertainty $g_{i i}$ ). These rapid changes are influenced by fading, which in turn deteriorates the performance of the controller, especially at higher Doppler frequencies (i.e., the upper bound $c_{2}$ in (14) and (24) has to be large to upper bound these effects). These rapid changes are exacerbated by
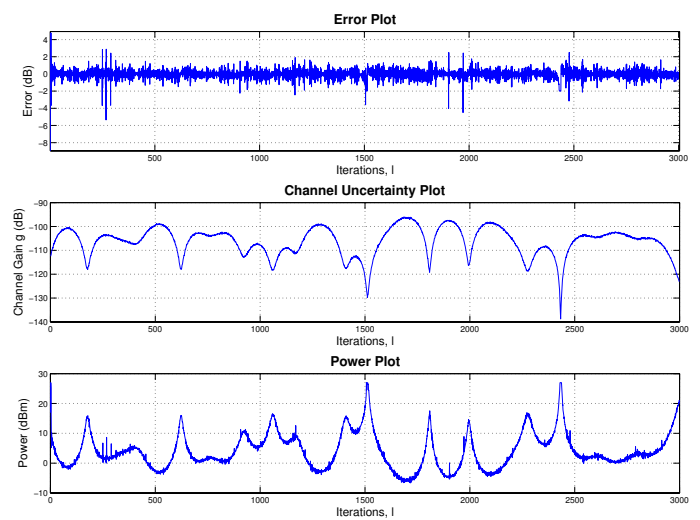

Fig. 3. Error, Channel gain and power plot: MT with a doppler frequency of $1.98 \mathrm{~Hz}$
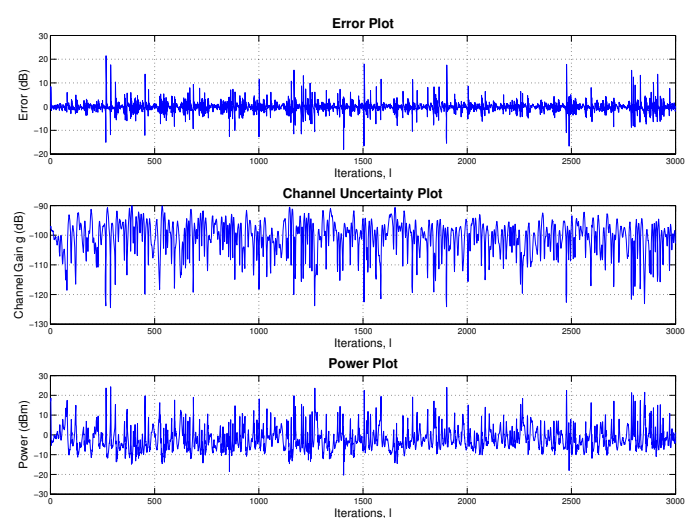

Fig. 4. Error, Channel gain and power plot: MT with a doppler frequency of $34.14 \mathrm{~Hz}$

MTs coming out of a deep faded zone (i.e., the channel gain is very close to zero) and the channel gain at the next sample can lead to high value causing interference to other users. Increasing $k_{n}$ can counter these effects (i.e., see (24)), but the magnitude of $k_{n}$ is limited by the power update law in (16) and the constraint that $0<P_{i}(l) \leq 500 \mathrm{~mW}$. In some other cases this phenomena is coupled with over-fading, when the power of some MTs reach an upper saturation limit and the controller can no longer increase the power to compensate for the fading.

\section{CONCLUSION}

A power controller is developed for a wireless cellular network system despite uncertain multi-channel fading. A Lyapunov-based analysis is used to develop an ultimate bound for the sampled SINR error which can be decreased up to a point by increasing a nonlinear damping gain. An analysis is also provided to illustrate how mobility and the desired SINR regulation range affects the choice of channel update times. Simulations indicate that the SINRs of most radio links are maintained in the desired communication 
TABLE I

PERCENTAGE OF SAMPLES WITHIN THE DESIRED SINR RANGE

\begin{tabular}{|l|l|l|}
\hline $\begin{array}{c}\text { Doppler } \\
\text { frequency }(\mathrm{Hz})\end{array}$ & $\begin{array}{c}\text { \% of samples where } \\
\gamma_{\min } \leq x_{i} \leq \gamma_{\max }\end{array}$ & $\begin{array}{c}\% \text { of samples where } \\
x_{i} \leq \gamma_{\min }\end{array}$ \\
\hline 1.98 & 98.97 & 0.23 \\
\hline 2.49 & 98.67 & 0.27 \\
\hline 3.25 & 99.23 & 0.40 \\
\hline 4.71 & 97.47 & 1.23 \\
\hline 6.35 & 97.63 & 0.83 \\
\hline 9.86 & 94.90 & 1.97 \\
\hline 10.84 & 95.07 & 2.47 \\
\hline 18.28 & 88.33 & 3.87 \\
\hline 18.36 & 88.86 & 5.13 \\
\hline 34.41 & 75.40 & 10.30 \\
\hline
\end{tabular}

range. Outages at some samples were determined to be due to limitations on power and on the predictive capability of the regulation controller, which highlight the need for future control development to address these issues.

\section{REFERENCES}

[1] J. Zander., "Optimum global transmitter power control in cellular radio systems," in IEEE Int. Symp. on Personal, Indoor and Mobile Radio Commun., London, U.K., 1991.

[2] — "Distributed cochannel interference control in cellular radio systems," IEEE Tran. Veh. Technol., vol. 41, pp. 305-311, 1992.

[3] _ , "Performance of optimum transmitter power control in cellular radio systems," IEEE Trans. Veh. Technol., vol. 41, pp. 57-62, 1992.

[4] G. Foschini and Z. Miljanic, "A simple distributed autonomous power control algorithm algorithm and its convergence," IEEE Tran. Veh. Technol., vol. 42, pp. 641-646, 1993.

[5] S. Grandhi and J. Zander, "Constrained power control in cellular radio systems," in Vehicular Technology Conference, 1994 IEEE 44th, 8-10 June 1994, pp. 824-828vol.2.

[6] R. D. Yates, "A framework for uplink power control in cellular radio systems," IEEE J. Select. Areas Commun., vol. 13, pp. 1341-1347, Sept. 1995

[7] R. Jantti and S. Kim., "Second-order power control with asymptotically fast convergence," IEEE J. Select. Commun, vol. 18, no. 3, 2000.

[8] N. Bambos, S. Chen, and G. Pottie., "Channel access algorithms with active link protection for wireless communication networks with power control," IEEE/ACM Trans. Networking, no. 5, pp. 583-597, 2000.

[9] J. Aein., "Power balancing in systems employing frequency reuse," COMSAT Tech. Rev., pp. 277-299, 1973.

[10] H. Alavi and R. Nettleton, "Downstream power control for a spread spectrum cellular mobile radio system," in Proc. IEEE GLOBECOM, 1982, pp. 84-88.

[11] S. Dontula and S. Jagannathan., "Active link protection for wireless peer-to-peer and cellular networks with power control," in World Wireless Congress, 2004, pp. 612-617.

[12] S. Jagannathan, Wireless Ad Hoc and Sensor Networks, F. L. Lewis, Ed. CRC Press, 2007.

[13] S. Jagannathan, M. Zawodniok, and Q. Shang, "Distributed power control for cellular networks in the presence of channel uncertainties," IEEE Transactions on Wireless Communications, vol. 5, no. 3, 2006.

[14] R. H. Clarke, "A statistical theory of mobile-radio reception," Bell Syst. Technical J., vol. 47, no. 6, pp. 957-1000, Jul--Aug. 1968.

[15] L. Gao and T. Wong, "Power control and spreading sequence allocation in a cdma forward link," IEEE Trans. Inform. Theory, vol. 50, no. 1, pp. 105-124, Jan. 2004.

[16] A.I-Osery and C. Abdallah., "Distributed power control in cdma cellular systems," IEEE Antennas Propagat. Mag., vol. 42, no. 4, 2000.

[17] N. Bambos., "Towards power-sensitive network architechtures in wireless communications: concepts, issues and design aspects," IEEE Pers. Commun., pp. 50-59, 1998

[18] T. Rappaport, Wireless Communications, Principles and Practice. Prentice Hall, 1999.
[19] J. Spooner, M. Maggiore, R. Ordonez, and K. Passino, Stable Adaptive Control and Estimation for Nonlinear Systems. Wiley, 2002.

[20] S. PalChaudhuri, J.-Y. Le Boudec, and M. Vojnovic, "Perfect simulations for random trip mobility models," in Simulation Symposium, 2005. Proceedings. 38th Annual, 4-6 April 2005, pp. 72-79.

[21] J.-Y. Le Boudec and M. Vojnovic, "Perfect simulation and stationarity of a class of mobility models," in INFOCOM 2005. 24th Annual Joint Conference of the IEEE Computer and Communications Societies. Proceedings IEEE, vol. 4, 13-17 March 2005, pp. 2743-2754vol.4.

[22] S. Kandukuri and S. Boyd, "Optimal power control in interferencelimited fading wireless channels with outage-probability specifications," IEEE Transactions on Wireless Communications, vol. 1, no. 1, pp. 46-55, January 2002.

[23] J. Papandriopoulos, J. Evans, and S. Dey, "Optimal power control for Rayleigh-faded multiuser systems with outage constraints," IEEE Transactions on Wireless Communications, vol. 4, no. 6, pp. 27052715, November 2005

\section{APPENDIX}

Taking the first difference of the SINR defined in (3) using (4), neglecting the residual terms, and approximating $\left(I_{i}(l)+\Delta I_{i}(l)\right) \approx I_{i}(l)$ yields

$$
x_{i}(l+1)=\alpha_{i}(l, x) x_{i}(l)+u_{i}(l),
$$

where

$$
\begin{aligned}
\alpha_{i}(l, x)= & a I_{i}^{-1}(l) P_{i}(l)\left[\frac{g_{i i}(l+1)}{x_{i}(l)}\right. \\
& -\sum_{i \neq j} \frac{\left(\Delta g_{i j}(l) P_{j}(l)\right)}{P_{i}(l)} \\
& \left.+\sum_{i \neq j} \frac{\left(g_{i j}(l) \Delta P_{j}(l)\right)}{P_{i}(l)}\right],
\end{aligned}
$$

and

$$
u_{i}(l)=\frac{x_{i}(l)}{P_{i}(l)}\left[P_{i}(l+1)-P_{i}(l)\right],
$$

since $\frac{a g_{i i}(l)}{I_{i}(l)}=\frac{x_{i}(l)}{P_{i}(l)}$. After including measurement noise, $\xi_{i}(l, x)$, the expression in (31) can be rewritten as

$$
x_{i}(l+1)=\alpha_{i}(l, x) x_{i}(l)+u_{i}(l)+\xi_{i}(l, x) .
$$

By defining the interference $I(l) \in \mathbb{R}^{n \times n}$ as a diagonal matrix with entries $I_{i}(l)$ expressed in (5), $g(l) \in \mathbb{R}^{n \times n}$ as a diagonal matrix with entries $g_{i i}(l)$, and $P(l) \in \mathbb{R}^{n}$, then the MIMO system in (1) can be developed, where $\alpha(l, x)=$ $\operatorname{diag}\left(\alpha_{i}(l, x)\right) \in \mathbb{R}^{n \times n}$, and $\xi(l, x), x(l), u(l) \in \mathbb{R}^{n}$. 